International Journal of Pure and Applied Mathematics

Volume 89 No. 4 2013, 565-581

ISSN: 1311-8080 (printed version); ISSN: 1314-3395 (on-line version)

url: http://www.ijpam.eu

doi: http://dx.doi.org/10.12732/ijpam.v89i4.11

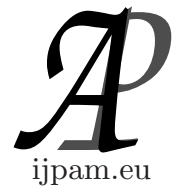

\title{
LAPLACIAN MINIMUM DOMINATING ENERGY OF A GRAPH
}

\author{
M.R. Rajesh Kanna ${ }^{1 \S}$, B.N. Dharmendra ${ }^{2}$, G. Sridhara ${ }^{3}$ \\ ${ }^{1,2,3}$ Post Graduate Department of Mathematics \\ Maharani's Science College for Women \\ J.L.B. Road, Mysore, 570 005, INDIA \\ ${ }^{3}$ Research Scholar \\ Research and Development Centre \\ Bharathiar University \\ Coimbatore, 641 046, INDIA
}

\begin{abstract}
Recently Prof.Chandrashekara adiga et al. [1] defined the minimum covering energy, $E_{C}(G)$ of a graph which depends on its particular minimum cover $C$. Motivated by this, we introduced minimum dominating energy [17] of a graph $E_{D}(G)$. In this paper we computed Laplacian minimum dominating energies of star graph, complete graph, crown graph and cocktail party graphs. Upper and lower bounds for $L E_{D}(G)$ are established.
\end{abstract}

AMS Subject Classification: 05C50, 05C69

Key Words: minimum dominating set, Laplacian minimum dominating matrix, Laplacian minimum dominating eigenvalues, Laplacian minimum dominating energy of a graph

\section{Introduction}

The concept of energy of a graph was introduced by I. Gutman [9] in 1978. Let $\mathrm{G}$ be a graph with $n$ vertices and $m$ edges and let $A=\left(a_{i j}\right)$ be the adjacency matrix of the graph. The eigenvalues $\lambda_{1}, \lambda_{2}, \cdots, \lambda_{n}$ of $\mathrm{A}$, assumed in non

Received: August 30, 2013

(C) 2013 Academic Publications, Ltd.

${ }^{\S}$ Correspondence author url: www.acadpubl.eu 
increasing order, are the eigenvalues of the graph G. As A is real symmetric, the eigenvalues of $\mathrm{G}$ are real with sum equal to zero. The energy $E(G)$ of $\mathrm{G}$ is defined to be the sum of the absolute values of the eigenvalues of G. i.e., $E(G)$ $=\sum_{i=1}^{n}\left|\lambda_{i}\right|$. For details on the mathematical aspects of the theory of graph energy see the reviews $[11,12]$ and the references cited therein. Studies on covering energy, maximum degree energy, minimum covering distance energy, dominating energies can be found in $[1,2,15,16,17]$ and the references cited there in.

I. Gutman and B. Zhou [10] defined the Laplacian energy of a graph G in the year 2006 . Let $\mathrm{G}$ be a graph with $n$ vertices and $m$ edges. The Laplacian matrix of the graph $\mathrm{G}$, denoted by $L=\left(L_{i j}\right)$, is a square matrix of order $\mathrm{n}$ whose elements are defined as

$$
L_{i j}= \begin{cases}-1 & \text { if } v_{i} \text { and } v_{j} \text { are adjacent, } \\ 0 & \text { if } v_{i} \text { and } v_{j} \text { are not adjacent, where } d_{i} \text { is the degree of the } \\ d_{i} & \text { if } i=j,\end{cases}
$$
vertex $v_{i}$. Let $\mu_{1}, \mu_{2}, \cdots, \mu_{n}$ be the Laplacian eigenvalues of G. Laplacian energy $\mathrm{LE}(\mathrm{G})$ of $\mathrm{G}$ is defined as $\mathrm{LE}(\mathrm{G})=\sum_{i=1}^{n}\left|\mu_{i}-\frac{2 m}{n}\right|$. The basic properties including various upper and lower bounds for Laplacian energy have been established in $[3,6,7,13,14,19,20,21,22,23,24,25,26,27]$ and it has found remarkable chemical applications, the molecular orbital theory of conjugated molecules [5].

\section{Definitions and Example}

\subsection{The Minimum Dominating Energy of a Graph}

Let $G$ be a simple graph of order $n$ with vertex set $V=\left\{v_{1}, v_{2}, \ldots, v_{n}\right\}$ and edge set E. A subset $D$ of $V$ is called a dominating set of $G$ if every vertex of $V-D$ is adjacent to some vertex in $D$. Any dominating set with minimum cardinality is called a minimum dominating set. Let $D$ be a minimum dominating set of a graph $G$. The minimum dominating matrix of $\mathrm{G}$ is the $n \times n$ matrix defined by $A_{D}(G):=\left(a_{i j}^{d}\right)$, where $a_{i j}^{d}= \begin{cases}1 & \text { if } v_{i} v_{j} \in E \\ 1 & \text { if } i=j \text { and } v_{i} \in D \\ 0 & \text { otherwise }\end{cases}$

The characteristic polynomial of $A_{D}(G)$ is denoted by $f_{n}(G, \lambda)=\operatorname{det}(\lambda I-$ $\left.A_{D}(G)\right)$. The minimum dominating eigenvalues of the graph $G$ are the eigen- 
values of $A_{D}(G)$. Since $A_{D}(G)$ is real and symmetric, its eigenvalues are real numbers and we label them in non-increasing order $\lambda_{1} \geq \lambda_{2} \geq \cdots \geq \lambda_{n}$. The minimum dominating energy of $\mathrm{G}$ is defined as $E_{D}(G):=\sum_{i=1}^{n}\left|\lambda_{i}\right|$.

Note that the trace of $A_{D}(G)=$ Domination Number $=k$.

\subsection{The Laplacian Minimum Domination Energy of a Graph}

Let $D(G)$ be the diagonal matrix of vertex degrees of the graph $\mathrm{G}$. Then $L_{D}(G)=D(G)-A_{D}(G)$ is called the Laplacian minimum dominating matrix of G. Let $\mu_{1}, \mu_{2}, \cdots, \mu_{n}$ be the eigenvalues of $L_{D}(G)$, arranged in non-increasing order . These eigenvalues are called Laplacian minimum dominating eigenvalues of $G$. The Laplacian minimum dominating energy of the graph $\mathrm{G}$ is defined as $L E_{D}(G):=\sum_{i=1}^{n}\left|\mu_{i}-\frac{2 m}{n}\right|$,where $m$ is the number of edges of $G$ and $\frac{2 m}{n}$ is the average degree of $G$.

In this paper, we are interested in studying mathematical aspects of the Laplacian minimum dominating energy of a graph. It is possible that the Laplacian minimum dominating energy that we are considering in this paper may have some applications in chemistry as well as in other areas.

Example 1. The possible minimum dominating sets for the following graph $G$ (see Figure 1) are i) $D_{1}=\left\{v_{1}, v_{5}\right\} \quad$ ii) $D_{2}=\left\{v_{2}, v_{5}\right\} \quad$ iii) $D_{3}=\left\{v_{2}, v_{6}\right\}$ i)If the dominating set is $D_{1}=\left\{v_{1}, v_{5}\right\}$ then

$$
\begin{aligned}
& A_{D_{1}}(G)=\left(\begin{array}{cccccc}
1 & 1 & 0 & 0 & 0 & 0 \\
1 & 0 & 1 & 1 & 1 & 0 \\
0 & 1 & 0 & 0 & 1 & 0 \\
0 & 1 & 0 & 0 & 1 & 0 \\
0 & 1 & 1 & 1 & 1 & 1 \\
0 & 0 & 0 & 0 & 1 & 0
\end{array}\right) \quad \text { and } \quad D(G)=\left(\begin{array}{cccccc}
1 & 0 & 0 & 0 & 0 & 0 \\
0 & 4 & 0 & 0 & 0 & 0 \\
0 & 0 & 2 & 0 & 0 & 0 \\
0 & 0 & 0 & 2 & 0 & 0 \\
0 & 0 & 0 & 0 & 4 & 0 \\
0 & 0 & 0 & 0 & 0 & 1
\end{array}\right) \text {, } \\
& L_{D_{1}}(G)=D(G)-A_{D_{1}}(G)=\left(\begin{array}{cccccc}
0 & -1 & 0 & 0 & 0 & 0 \\
-1 & 4 & -1 & -1 & -1 & 0 \\
0 & -1 & 2 & 0 & -1 & 0 \\
0 & -1 & 0 & 2 & -1 & 0 \\
0 & -1 & -1 & -1 & 3 & -1 \\
0 & 0 & 0 & 0 & -1 & 1
\end{array}\right)
\end{aligned}
$$




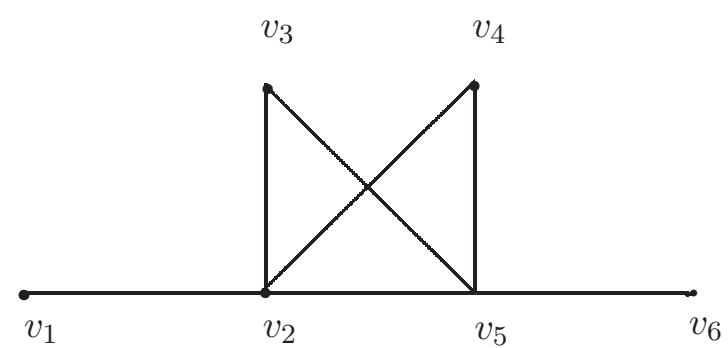

Figure 1

Characteristic equation is

$$
\mu^{6}-12 \mu^{5}+48 \mu^{4}-69 \mu^{3}+16 \mu^{2}+22 \mu-4=0 .
$$

The Laplacian dominating eigenvalues are $\mu_{1} \approx-0.4856, \mu_{2} \approx 0.1744, \mu_{3} \approx$ $1.1279, \mu_{4} \approx 2, \mu_{5} \approx 4.2080, \mu_{6} \approx 4.9754$.

Average degree $=\frac{2 m}{n}=\frac{2 \times 7}{6}=\frac{7}{3}$

Hence Laplacian minimum dominating energy, $L E_{D_{1}}(G) \approx 11.0334$.

ii) If the dominating set is $D_{2}=\left\{v_{2}, v_{5}\right\}$ then

$$
\begin{aligned}
A_{D_{2}}(G)= & \left(\begin{array}{cccccc}
0 & 1 & 0 & 0 & 0 & 0 \\
1 & 1 & 1 & 1 & 1 & 0 \\
0 & 1 & 0 & 0 & 1 & 0 \\
0 & 1 & 0 & 0 & 1 & 0 \\
0 & 1 & 1 & 1 & 1 & 1 \\
0 & 0 & 0 & 0 & 1 & 0
\end{array}\right), \\
L_{D_{2}}(G)=D(G)-A_{D_{2}}(G) & =\left(\begin{array}{cccccccc}
1 & -1 & 0 & 0 & 0 & 0 \\
-1 & 3 & -1 & -1 & -1 & 0 \\
0 & -1 & 2 & 0 & -1 & 0 \\
0 & -1 & 0 & 2 & -1 & 0 \\
0 & -1 & -1 & -1 & 3 & -1 \\
0 & 0 & 0 & 0 & -1 & 1
\end{array}\right) .
\end{aligned}
$$

Characteristic equation is $\mu^{6}-12 \mu^{5}+51 \mu^{4}-90 \mu^{3}+55 \mu^{2}+8 \mu-12=0$. 
The Laplacian minimum dominating eigenvalues are $\mu_{1} \approx-0.3914, \mu_{2} \approx$ $0.6972, \mu_{3} \approx 1.2271, \mu_{4} \approx 2, \mu_{5} \approx 4.1642, \mu_{6} \approx 4.3028$.

$\therefore$ Laplacian minimum dominating energy, $L E_{D_{2}}(G) \approx 9.6007$.

Thus Laplacian minimum dominating energy of graph $G$ depends on the dominating set.

\section{Laplacian Minimum Dominating Energy of Some Standard Graphs}

Theorem 3.1. For $n \geq 2$, the Laplacian minimum dominating energy of a Star graph $K_{1, n-1}$ is $\frac{(n-2)^{2}}{n}+\sqrt{n^{2}-2 n+5}$.

Proof. Consider a Star graph $K_{1, n-1}$ with vertex set $V=\left\{v_{0}, v_{1}, v_{2}, \ldots, v_{n-1}\right\}$ and center $v_{0}$. Then minimum dominating set is $D=\left\{v_{0}\right\}$,

$$
A_{D}\left(K_{1, n-1}\right)=\left(\begin{array}{ccccc}
1 & 1 & 1 & \ldots & 1 \\
1 & 0 & 0 & \ldots & 0 \\
1 & 0 & 0 & \ldots & 0 \\
\vdots & \vdots & \vdots & \ddots & \vdots \\
1 & 0 & 0 & \ldots & 0
\end{array}\right)_{n \times n}
$$

and

$$
\begin{gathered}
D\left(K_{1, n-1}\right)=\left(\begin{array}{ccccc}
n-1 & 0 & 0 & \ldots & 0 \\
0 & 1 & 0 & \ldots & 0 \\
0 & 0 & 1 & \ldots & 0 \\
\vdots & \vdots & \vdots & \ddots & \vdots \\
0 & 0 & 0 & \ldots & 1
\end{array}\right)_{n \times n} \\
\therefore L_{D}\left(K_{1, n-1}\right)=D\left(K_{1, n-1}\right)-A_{D}\left(K_{1, n-1}\right)=\left(\begin{array}{cccccc}
n-2 & -1 & -1 & \ldots & -1 \\
-1 & 1 & 0 & \ldots & 0 \\
-1 & 0 & 1 & \ldots & 0 \\
\vdots & \vdots & \vdots & \ddots & \vdots \\
-1 & 0 & 0 & \ldots & 1
\end{array}\right)_{n \times n}
\end{gathered}
$$

Characteristic equation is

$$
(\mu-1)^{(n-2)}\left(\mu^{2}-(n-1) \mu-1\right)=0 .
$$


Laplacian minimum dominating eigenvalues are

$$
\mu=1(n-2 \text { times }), \quad \mu=\frac{(n-1) \pm \sqrt{n^{2}-2 n+5}}{2}
$$

(one time each) Number of vertices $=n$, Number of edges $=n-1$.

$\therefore$ Average degree $=\frac{2(n-1)}{n}$.

Hence Laplacian minimum dominating energy,

$$
\begin{aligned}
L E_{D}\left(K_{1, n-1}\right)=\mid & -\frac{2(n-1)}{n}|(n-2)+| \frac{(n-1)+\sqrt{n^{2}-2 n+5}}{2}-\frac{2(n-1)}{n} \mid \\
+ & \left|\frac{(n-1)-\sqrt{n^{2}-2 n+5}}{2}-\frac{2(n-1)}{n}\right| \\
=\left|\frac{-n+2}{n}\right|(n-2)+\left|\frac{n^{2}-n+n \sqrt{n^{2}-2 n+5}-4 n+4}{2 n}\right| & \quad\left|\frac{n^{2}-n-n \sqrt{n^{2}-2 n+5}-4 n+4}{2 n}\right| \\
= & \frac{(n-2)(n-2)}{n}+\left|\frac{n^{2}-5 n+4+n \sqrt{n^{2}-2 n+5}}{2 n}\right| \\
\quad+\left|\frac{n^{2}-5 n+4-n \sqrt{n^{2}-2 n+5}}{2 n}\right| & \frac{(n-2)^{2}}{n}+\sqrt{n^{2}-2 n+5} .
\end{aligned}
$$

Theorem 3.2. For $n \geq 2$, the Laplacian minimum dominating energy of Complete graph $K_{n}$ is $(n-2)+\sqrt{n^{2}-2 n+5}$.

Proof. Consider the complete graph $K_{n}$ with vertex set $V=\left\{v_{1}, v_{2}, \ldots, v_{n}\right\}$. The minimum dominating set is $D=\left\{v_{1}\right\}$.

$$
A_{D}\left(K_{n}\right)=\left(\begin{array}{cccccc}
1 & 1 & 1 & \ldots & 1 & 1 \\
1 & 0 & 1 & \ldots & 1 & 1 \\
1 & 1 & 0 & \ldots & 1 & 1 \\
\vdots & \vdots & \vdots & \ddots & \vdots & \vdots \\
1 & 1 & 1 & \ldots & 0 & 1 \\
1 & 1 & 1 & \ldots & 1 & 0
\end{array}\right)_{n \times n}
$$




$$
\begin{aligned}
D\left(K_{n}\right) & =\left(\begin{array}{ccccccc}
n-1 & 0 & 0 & \ldots & 0 & \\
0 & n-1 & 0 & \ldots & 0 & \\
0 & 0 & n-1 & \ldots & 0 \\
\vdots & \vdots & \vdots & \ddots & \vdots \\
0 & 0 & 0 & \ldots & n-1
\end{array}\right)_{n \times n} \\
\therefore L_{D}\left(K_{n}\right)=D\left(K_{n}\right) & -A_{D}\left(K_{n}\right) \\
& =\left(\begin{array}{ccccccc}
n-2 & -1 & -1 & \ldots & -1 & -1 \\
-1 & n-1 & -1 & \ldots & -1 & -1 \\
-1 & -1 & n-1 & \ldots & -1 & -1 \\
\vdots & \vdots & \vdots & \ddots & \vdots & \vdots \\
-1 & -1 & -1 & \ldots & n-1 & -1 \\
-1 & -1 & -1 & \ldots & -1 & n-1
\end{array}\right)_{n \times n} .
\end{aligned}
$$

Characteristic equation is

$$
[\mu-n)]^{(n-2)}\left[\mu^{2}-(n-1) \mu-1\right]=0 .
$$

Laplacian minimum dominating eigenvalues are $\mu=n[(\mathrm{n}-2)$ times $]$ and $\mu=\frac{(n-1) \pm \sqrt{n^{2}-2 n+5}}{2} \quad$ [one time each]

Number of vertices $=n$, Number of edges $=n C_{2}=\frac{n(n-1)}{2}$

$\therefore$ Average degree $=\frac{2 m}{n}=2 \frac{\frac{n(n-1)}{2}}{n}=n-1$

Laplacian minimum dominating energy, $L E_{D}\left(K_{n}\right)$

$$
\begin{aligned}
= & |n-(n-1)|(n-2)+\left|\frac{(n-1)+\sqrt{n^{2}-2 n+5}}{2}-(n-1)\right| \\
& +\left|\frac{(n-1)-\sqrt{n^{2}-2 n+5}}{2}-(n-1)\right| \\
= & (n-2)+\left|\frac{-n+1+\sqrt{n^{2}-2 n+5}}{2}\right|+\left|\frac{-n+1-\sqrt{n^{2}-2 n+5}}{2}\right| \\
= & (n-2)+\sqrt{n^{2}-2 n+5} .
\end{aligned}
$$

Theorem 3.3. For $n \geq 2$, the Laplacian minimum dominating energy of Crown graph $S_{n}^{0}$ is $(2 n-4)+\sqrt{n^{2}-2 n+5}+\sqrt{n^{2}+2 n-3}$. 
Proof. Consider the Crown graph $S_{n}^{0}$ with vertex set $V=\left\{u_{1}, u_{2}, \ldots, u_{n}, v_{1}\right.$, $\left.v_{2}, \ldots, v_{n}\right\}$. The minimum dominating set is $D=\left\{u_{1}, v_{1}\right\}$.

$$
\begin{aligned}
& A_{D}\left(S_{n}^{0}\right)=\left(\begin{array}{cccccccccc}
1 & 0 & 0 & \ldots & 0 & 0 & 1 & 1 & \ldots & 1 \\
0 & 0 & 0 & \ldots & 0 & 1 & 0 & 1 & \ldots & 1 \\
0 & 0 & 0 & \ldots & 0 & 1 & 1 & 0 & \ldots & 1 \\
\vdots & \vdots & \vdots & \ddots & \vdots & \vdots & \vdots & \vdots & \ddots & \vdots \\
0 & 0 & 0 & \ldots & 0 & 1 & 1 & 1 & \ldots & 0 \\
0 & 1 & 1 & \ldots & 1 & 1 & 0 & 0 & \ldots & 0 \\
1 & 0 & 1 & \ldots & 1 & 0 & 0 & 0 & \ldots & 0 \\
1 & 1 & 0 & \ldots & 1 & 0 & 0 & 0 & \ldots & 0 \\
\vdots & \vdots & \vdots & \ddots & \vdots & \vdots & \vdots & \vdots & \ddots & \vdots \\
1 & 1 & 1 & \ldots & 0 & 0 & 0 & 0 & \ldots & 0
\end{array}\right)_{2 n \times 2 n} \\
& D\left(S_{n}^{0}\right)=\left(\begin{array}{ccccc}
n-1 & 0 & 0 & \ldots & 0 \\
0 & n-1 & 0 & \ldots & 0 \\
0 & 0 & n-1 & \ldots & 0 \\
\vdots & \vdots & \vdots & \ddots & \vdots \\
0 & 0 & 0 & \ldots & n-1
\end{array}\right)_{2 n \times 2 n} \\
& L_{D}\left(S_{n}^{0}\right)=D\left(S_{n}^{0}\right)-A_{D}\left(S_{n}^{0}\right) \\
& L_{\mathrm{D}}\left(S_{\mathrm{n}}^{0}\right)=\left(\begin{array}{cccccccccc}
n-2 & 0 & 0 & \ldots & 0 & 0 & -1 & -1 & \ldots & -1 \\
0 & n-1 & 0 & \ldots & 0 & -1 & 0 & -1 & \cdots & -1 \\
0 & 0 & n-1 & \cdots & 0 & -1 & -1 & 0 & \cdots & -1 \\
\vdots & \vdots & \vdots & \ddots & \vdots & \vdots & \vdots & \vdots & \ddots & \vdots \\
0 & 0 & 0 & \ldots & n-1 & -1 & -1 & -1 & \ldots & 0 \\
0 & -1 & -1 & \ldots & -1 & n-2 & 0 & 0 & \cdots & 0 \\
-1 & 0 & -1 & \ldots & -1 & 0 & n-1 & 0 & \ldots & 0 \\
-1 & -1 & 0 & \cdots & -1 & 0 & 0 & n-1 & \cdots & 0 \\
\vdots & \vdots & \vdots & \ddots & \vdots & \vdots & \vdots & \vdots & \ddots & \vdots \\
-1 & -1 & -1 & \cdots & 0 & 0 & 0 & 0 & \cdots & n-1
\end{array}\right)_{2 n \times 2 n}
\end{aligned}
$$

Characteristic equation is

$$
[\mu-n]^{n-2}[\mu-n+2]^{n-2}\left[\mu^{2}-(n-1) \mu-1\right]\left[\mu^{2}-(3 n-5) \mu+\left(2 n^{2}-8 n+7\right)\right]=0
$$

Laplacian minimum dominating eigenvalues are $\mu=n[(\mathrm{n}-2)$ times $], \quad \mu=n-2[(\mathrm{n}-2)$ times $]$ $\mu=\frac{(n-1) \pm \sqrt{n^{2}-2 n+5}}{2}\left[\right.$ one time each], $\mu=\frac{(3 n-5) \pm \sqrt{n^{2}+2 n-3}}{2}$ [one time each].

Number of vertices $=2 n$, Number of edges $=n(n-1)$, 
$\therefore$ Average degree $=\frac{2 n(n-1)}{2 n}=n-1$.

Laplacian minimum dominating energy,

$$
\begin{array}{r}
L E_{D}\left(S_{n}^{0}\right)=|n-(n-1)|(n-2)+|(n-2)-(n-1)|(n-2) \\
+\left|\frac{n-1+\sqrt{n^{2}-2 n+5}}{2}-(n-1)\right|+\left|\frac{n-1-\sqrt{n^{2}-2 n+5}}{2}-(n-1)\right| \\
+\left|\frac{3 n-5+\sqrt{n^{2}+2 n-3}}{2}-(n-1)\right|+\left|\frac{3 n-5-\sqrt{n^{2}+2 n-3}}{2}-(n-1)\right| \\
=(n-2)+(n-2)+\left|\frac{-n+1+\sqrt{n^{2}-2 n+5}}{2}\right|+\left|\frac{-n+1-\sqrt{n^{2}-2 n+5}}{2}\right| \\
+\left|\frac{n-3+\sqrt{n^{2}+2 n-3}}{2}\right|+\left|\frac{n-3-\sqrt{n^{2}+2 n-3}}{2}\right| \\
=(2 n-4)+\sqrt{n^{2}-2 n+5}+\sqrt{n^{2}+2 n-3} .
\end{array}
$$

Theorem 3.4. The Laplacian minimum dominating energy of Cocktail party graph $K_{n \times 2}$ is $(2 n-3)+\sqrt{4 n^{2}-4 n+9}$.

Proof. Consider Cocktail party graph $K_{n \times 2}$ with vertex set $\mathrm{V}=\bigcup_{i=1}^{n-1}\left\{u_{i}, v_{i}\right\}$ .The minimum dominating set is $D=\left\{u_{1}, v_{1}\right\}$.

$$
\begin{aligned}
& A_{D}\left(K_{n \times 2}\right)=\left(\begin{array}{ccccccccc}
1 & 0 & 1 & 1 & \ldots & 1 & 1 & 1 & 1 \\
0 & 1 & 1 & 1 & \ldots & 1 & 1 & 1 & 1 \\
1 & 1 & 0 & 0 & \ldots & 1 & 1 & 1 & 1 \\
1 & 1 & 0 & 0 & \ldots & 1 & 1 & 1 & 1 \\
\vdots & \vdots & \vdots & \vdots & \ddots & \vdots & \vdots & \vdots & \vdots \\
1 & 1 & 1 & 1 & \ldots & 0 & 0 & 1 & 1 \\
1 & 1 & 1 & 1 & \ldots & 0 & 0 & 1 & 1 \\
1 & 1 & 1 & 1 & \ldots & 1 & 1 & 0 & 0 \\
1 & 1 & 1 & 1 & \ldots & 1 & 1 & 0 & 0
\end{array}\right)_{2 n \times 2 n} \\
& D\left(K_{n \times 2}\right)=\left(\begin{array}{cccccc}
2(n-1) & 0 & 0 & \ldots & 0 & 0 \\
0 & 2(n-1) & 0 & \ldots & 0 & 0 \\
0 & 0 & 2(n-1) & \ldots & 0 & 0 \\
\vdots & \vdots & \vdots & \ddots & \vdots & \vdots \\
0 & 0 & 0 & \ldots & 2(n-1) & 0 \\
0 & 0 & 0 & \ldots & 0 & 2(n-1)
\end{array}\right)_{2 n \times 2 n}
\end{aligned}
$$




$$
L_{D}\left(K_{n \times 2}\right)=D\left(K_{n \times 2}\right)-A_{D}\left(K_{n \times 2}\right)
$$

$$
L_{\mathrm{D}}\left(K_{\mathrm{n}} \times 2\right)=\left(\begin{array}{ccccccccc}
2 n-3 & 0 & -1 & -1 & \cdots & -1 & -1 & -1 & -1 \\
0 & 2 n-3 & -1 & -1 & \cdots & -1 & -1 & -1 & -1 \\
-1 & -1 & 2 n-2 & 0 & \cdots & -1 & -1 & -1 & -1 \\
-1 & -1 & 0 & 2 n-2 & \cdots & -1 & -1 & -1 & -1 \\
\vdots & \vdots & \vdots & \vdots & \ddots & \vdots & \vdots & \vdots & \vdots \\
-1 & -1 & -1 & -1 & \cdots & 2 n-2 & 0 & -1 & -1 \\
-1 & -1 & -1 & -1 & \cdots & 0 & 2 n-2 & -1 & -1 \\
-1 & -1 & -1 & -1 & \cdots & -1 & -1 & 2 n-2 & 0 \\
-1 & -1 & -1 & -1 & \cdots & -1 & -1 & 0 & 2 n-2
\end{array}\right)_{2 n \times 2 n}
$$

Characteristic equation is

$$
(\mu-2 n+3)(\mu-2 n+2)^{n-1}(\mu-2 n)^{n-2}\left(\mu^{2}-(2 n-1) \mu-2\right)=0
$$

Laplacian minimum dominating eigenvalues are

$\mu=2 n-3$ [One time], $\mu=2 n-2$ [(n-1) times], $\mu=2 n$ [(n-2) times], $\mu=\frac{(2 n-1) \pm \sqrt{4 n^{2}-4 n+7}}{2}$ [one time each].

Number of vertices $=2 n, \quad$ Number of edges $=2 n(n-1)$

$\therefore$ Average degree $=\frac{2(2 n)(n-1)}{2 n}=2(n-1)$

Laplacian minimum dominating energy, $L E_{D}\left(K_{n \times 2}\right)$

$$
\begin{aligned}
& \quad=|(2 n-3)-(2 n-2)|+|(2 n-2)-(2 n-2)|(n-1)+|2 n-(2 n-2)|(n-2) \\
& +\left|\frac{2 n-1+\sqrt{4 n^{2}-4 n+9}}{2}-(2 n-2)\right|+\left|\frac{2 n-1-\sqrt{4 n^{2}-4 n+9}}{2}-(2 n-2)\right| \\
& =1+0+2 n-4++\left|\frac{-2 n+3+\sqrt{4 n^{2}-4 n+9}}{2}\right|+\left|\frac{-2 n+3-\sqrt{4 n^{2}-4 n+9}}{2}\right| \\
& =(2 n-3)+\sqrt{4 n^{2}-4 n+9} .
\end{aligned}
$$

\section{Properties of Laplacian Minimum Dominating Eigen Values of a Graph}

Theorem 4.1. If $D$ is a minimum dominating set of a graph $G$ and $\mu_{1}, \mu_{2}, \mu_{3}, \ldots, \mu_{n}$ are the eigenvalue of $L_{D}(G)$ then (i) $\sum_{i=1}^{n} \mu_{i}=2|E|-|D|$ and (ii) $\sum_{i=1}^{n} \mu_{i}^{2}=2|E|+\sum_{i=1}^{n}\left(d_{i}-c_{i}\right)^{2}$ where $c_{i}= \begin{cases}1 & \text { if } v_{i} \in D \\ 0 & \text { if } v_{i} \notin D\end{cases}$ 
Proof. (i) By definition, the sum of the principal diagonal elements of $L_{D}(G)$ is equal to $\sum_{i=1}^{n} d_{i}-|D|=2|E|-|D|$.

Also the sum of eigenvalues of $L_{D}(G)$ is trace of $L_{D}(G)$

$\therefore \sum_{i=1}^{n} \mu_{i}=2|E|-|D|$.

(ii) The sum of squares of eigenvalues of $L_{D}(G)$ is the trace of $L_{D}(G)^{2}$

$$
\begin{aligned}
\therefore \sum_{i=1}^{n} \mu_{i}^{2}=\sum_{i=1}^{n} \sum_{j=1}^{n} l_{i j}^{d} l_{j i}^{d} & =\sum_{i \neq j}\left(l_{i j}^{d}\right)^{2}+\sum_{i=1}^{n}\left(l_{i i}^{d}\right)^{2} \\
& =2 \sum_{i<j}\left(l_{i j}^{d}\right)^{2}+\sum_{i=1}^{n}\left(l_{i i}^{d}\right)^{2} \\
& =2|E|+\sum_{i=1}^{n}\left(d_{i}-c_{i}\right)^{2} \text { where } c_{i}= \begin{cases}1 & \text { if } v_{i} \in D \\
0 & \text { if } v_{i} \notin D\end{cases} \\
& =2 \mathrm{M}, \text { where } \mathrm{M}=|E|+\frac{1}{2} \sum_{i=1}^{n}\left(d_{i}-c_{i}\right)^{2}
\end{aligned}
$$

R. B. Bapat and Sukunta Pati [4] have shown that if the energy of a graph is rational then it must be an even integer. The similar result on absolute Laplacian minimum dominating eigenvalues is given below.

Theorem 4.2. Let $G$ be a graph with a minimum dominating set $D$. If the sum of the absolute values of Laplacian minimum dominating eigenvalues is a rational number, then it will be an integer satisfying $\sum_{i=1}^{n}\left|\mu_{i}\right| \equiv|D|(\bmod 2)$.

Proof. Let $\mu_{1}, \mu_{2}, \ldots, \mu_{n}$ be Laplacian minimum dominating eigenvalues of a graph $G$, of which $\mu_{1}, \mu_{2}, \ldots, \mu_{r}$ are positive and the rest are non-positive, then

$$
\begin{aligned}
\sum_{i=1}^{n}\left|\mu_{i}\right| & =\left(\mu_{1}+\mu_{2}+\ldots+\mu_{r}\right)-\left(\mu_{r+1}+\ldots+\mu_{n}\right) \\
\sum_{i=1}^{n}\left|\mu_{i}\right| & =2\left(\mu_{1}+\mu_{2}+\ldots+\mu_{r}\right)-\left(\mu_{1}+\mu_{2}+\ldots+\mu_{n}\right) \\
& =2\left(\mu_{1}+\mu_{2}+\ldots+\mu_{r}\right)-\sum_{i=1}^{n} \mu_{i} \\
& =2\left(\mu_{1}+\mu_{2}+\ldots+\mu_{r}\right)-(2|E|-|D|) \\
& =2\left(\mu_{1}+\mu_{2}+\ldots+\mu_{r}-|E|\right)+|D|
\end{aligned}
$$


By the result of Fiedler on additive compounds[8], the partial sum $\mu_{1}+$ $\mu_{2}+\ldots+\mu_{r}$ is an eigenvalue of a matrix whose characteristic polynomial has integer coefficients. If $\sum_{i=1}^{n}\left|\mu_{i}\right|$ is rational then $\mu_{1}+\mu_{2}+\ldots+\mu_{r}$ is rational and hence it must be an integer.

$$
\therefore \sum_{i=1}^{n}\left|\mu_{i}\right| \equiv|D|(\bmod 2)
$$

\section{Bounds on Laplacian Minimum Dominating Energy of a Graph}

Theorem 5.1. (Upper bound) Let $G$ be a graph with $n$ vertices, $m$ edges and $D$ is a minimum dominating set of a graph $G$.Then $L E_{D}(G) \leq \sqrt{2 M n}+2 m$.

Proof. Cauchy-Schwarz inequality is $\left(\sum_{i=1}^{n} a_{i} b_{i}\right)^{2} \leq\left(\sum_{i=1}^{n} a_{i}^{2}\right)\left(\sum_{i=1}^{n} b_{i}^{2}\right)$

$$
\begin{gathered}
\text { Put } a_{i}=1, b_{i}=\left|\mu_{i}\right| \text { then }\left(\sum_{i=1}^{n}\left|\mu_{i}\right|\right)^{2} \leq\left(\sum_{i=1}^{n} 1\right)\left(\sum_{i=1}^{n}\left|\mu_{i}\right|^{2}\right) \\
\text { i.e., }\left(\sum_{i=1}^{n}\left|\mu_{i}\right|\right)^{2} \leq \mathrm{n} 2 \mathrm{M} \\
\therefore \sum_{i=1}^{n}\left|\mu_{i}\right| \leq \sqrt{2 M n} .
\end{gathered}
$$

By Triangle inequality, $\left|\mu_{i}-\frac{2 m}{n}\right| \leq\left|\mu_{i}\right|+\left|\frac{2 m}{n}\right| \forall i=1,2 \ldots n$

$$
\begin{gathered}
\text { i.e., }\left|\mu_{i}-\frac{2 m}{n}\right| \leq\left|\mu_{i}\right|+\frac{2 m}{n} \quad \forall i \\
\begin{aligned}
\Rightarrow \sum_{i=1}^{n}\left|\mu_{i}-\frac{2 m}{n}\right| & \leq \sum_{i=1}^{n}\left|\mu_{i}\right|+\sum_{i=1}^{n} \frac{2 m}{n} \\
& \leq \sqrt{2 M n}+2 \mathrm{~m} \\
\therefore L E_{D}(G) & \leq \sqrt{2 M n}+2 \mathrm{~m}
\end{aligned}
\end{gathered}
$$

This bound is similar to B.J.McClelland's bound [18] for ordinary energy of graph. 
Theorem 5.2. (Upper bound)Let $G$ be a graph with $n$ vertices, $m$ edges and $D$ is a minimum dominating set of $G$. Then

$$
L E_{D}(G) \leq \sqrt{2 M n+4 m(|D|-m)} .
$$

Proof.

Cauchy- Schwarz inequality is $\left(\sum_{i=1}^{n} a_{i} b_{i}\right)^{2} \leq\left(\sum_{i=1}^{n} a_{i}^{2}\right)\left(\sum_{i=1}^{n} b_{i}^{2}\right)$

$$
\begin{aligned}
& \text { Put } a_{i}=1, b_{i}=\left|\mu_{i}-\frac{2 m}{n}\right| \text { then } \\
& \qquad \begin{aligned}
\left(\sum_{i=1}^{n}\left|\mu_{i}-\frac{2 m}{n}\right|\right)^{2} & \leq\left(\sum_{i=1}^{n} 1\right)\left(\sum_{i=1}^{n}\left|\mu_{i}-\frac{2 m}{n}\right|^{2}\right) \\
i . e .,\left[L E_{D}(G)\right]^{2} & =n\left[\sum_{i=1}^{n} \mu_{i}^{2}+\sum_{i=1}^{n} \frac{4 m^{2}}{n^{2}}-\frac{4 m}{n} \sum_{i=1}^{n} \mu_{i}\right] \\
& =n\left[2 M+\frac{4 m^{2}}{n^{2}} \cdot n-\frac{4 m}{n}(2 m-|D|)\right] \\
& =n\left[2 M+\frac{4 m^{2}}{n}-\frac{8 m^{2}}{n}+\frac{4 m|D|}{n}\right] \\
& =2 M n+4 m(|D|-m) \\
\therefore L E_{D}(G) & \leq \sqrt{2 M n+4 m(|D|-m) .}
\end{aligned}
\end{aligned}
$$

Theorem 5.3. (Lower bound): Let $G$ be a graph with $n$ vertices and $m$ edges and $D$ is a minimum dominating set of $G$. If $D=\left|\operatorname{det} L_{D}(G)\right|$ then $L E_{D}(G) \geq \sqrt{2 M+n(n-1) D^{\frac{2}{n}}}-2 m$.

$$
\begin{aligned}
\text { Proof. Consider }\left[\sum_{i=1}^{n}\left|\mu_{i}\right|\right]^{2} \\
=\left(\sum_{i=1}^{n}\left|\mu_{i}\right|\right) \cdot\left(\sum_{j=1}^{n}\left|\mu_{j}\right|\right) \\
=\sum_{i=1}^{n}\left|\mu_{i}\right|^{2}+\sum_{i \neq j}\left|\mu_{i} \| \mu_{j}\right|
\end{aligned}
$$




$$
\therefore \sum_{i \neq j}\left|\mu_{i}\right|\left|\mu_{j}\right|=\left(\sum_{i=1}^{n}\left|\mu_{i}\right|\right)^{2}-\sum_{i=1}^{n}\left|\mu_{i}\right|^{2}
$$

Applying inequality between the arithmetic and geometric means for $n(n-$ 1) terms, we have

$$
\begin{aligned}
& \frac{\sum_{i \neq j}\left|\mu_{i} \| \mu_{j}\right|}{n(n-1)} \geq\left[\prod_{i \neq j}\left|\mu_{i}\right|\left|\mu_{j}\right|\right]^{\frac{1}{n(n-1)}} \\
& \text { i.e., } \sum_{i \neq j}\left|\mu_{i}\right|\left|\mu_{j}\right| \geq n(n-1)\left[\prod_{i \neq j}\left|\mu_{i}\right|\left|\mu_{j}\right|\right]^{\frac{1}{n(n-1)}}
\end{aligned}
$$

Using (5.1) we get,

$$
\begin{gathered}
\left(\sum_{i=1}^{n}\left|\mu_{i}\right|\right)^{2}-\sum_{i=1}^{n}\left|\mu_{i}\right|^{2} \geq n(n-1)\left[\prod_{i=1}^{n}\left|\mu_{i}\right|^{2(n-1)}\right] \frac{1}{n(n-1)} \\
\left(\sum_{i=1}^{n}\left|\mu_{i}\right|\right)^{2}-2 M \geq n(n-1)\left[\prod_{i=1}^{n}\left|\mu_{i}\right|\right]^{\frac{2}{n}} \\
\left(\sum_{i=1}^{n}\left|\mu_{i}\right|\right)^{2} \geq 2 M+n(n-1)\left[\prod_{i=1}^{n} \mu_{i}\right]^{\frac{2}{n}} \\
\therefore \sum_{i=1}^{n}\left|\mu_{i}\right| \geq \sqrt{2 M+n(n-1) D^{\frac{2}{n}}}
\end{gathered}
$$

We know that $\left|\mu_{i}\right|-\left|\frac{2 m}{n}\right| \leq\left|\mu_{i}-\frac{2 m}{n}\right| \forall i$

$$
\begin{aligned}
\text { i.e., }\left|\mu_{i}\right|-\frac{2 m}{n} & \leq\left|\mu_{i}-\frac{2 m}{n}\right| \quad \forall i \\
\sum_{i=1}^{n}\left|\mu_{i}\right|-\sum_{i=1}^{n} \frac{2 m}{n} & \leq \sum_{i=1}^{n}\left|\mu_{i}-\frac{2 m}{n}\right| \\
\text { i.e., } \sum_{i=1}^{n}\left|\mu_{i}\right|-2 m & \leq L E_{D}(G) \\
\text { i.e., } L E_{D}(G) & \geq \sum_{i=1}^{n}\left|\mu_{i}\right|-2 m
\end{aligned}
$$




$$
\begin{aligned}
& \geq \sqrt{2 M+n(n-1) D^{\frac{2}{n}}}-2 m \\
\therefore L E_{D}(G) & \geq \sqrt{2 M+n(n-1) D^{\frac{2}{n}}}-2 m
\end{aligned}
$$

Theorem 5.4. Let $G$ be a graph with $n$ vertices, $m$ edges and $D$ is a minimum dominating set of $G$. If the sum of the absolute Laplacian minimum dominating eigenvalues is a rational number, then $L E_{D}(G) \in(|D|+2 t-2 m, \mid$ $D \mid+2 t+2 m)$, where $t$ is any integer such that $\sum_{i=1}^{n}\left|\mu_{i}\right| \equiv|D|(\bmod 2)$.

Proof.

$$
\begin{aligned}
& \text { We know that } \begin{aligned}
\sum_{i=1}^{n}\left|\mu_{i}-\frac{2 m}{n}\right| & \leq \sum_{i=1}^{n}\left|\mu_{i}\right|+2 m \\
\text { i.e., } L E_{D}(G) & \leq \sum_{i=1}^{n}\left|\mu_{i}\right|+2 m \\
& =|D|+2 t+2 m \\
\text { Also, } L E_{D}(G) & \geq \sum_{i=1}^{n}\left|\mu_{i}\right|-2 m \\
& =|D|+2 t-2 m \quad \text { From } \\
\therefore L E_{D}(G) & (|D|+2 t-2 m,|D|+2 t+2 m)
\end{aligned}
\end{aligned}
$$

\section{References}

[1] C. Adiga, A. Bayad, I. Gutman, S. A. Srinivas, The minimum covering energy of a graph.Kragujevac J. Sci. 34 (2012) 39-56.

[2] C. Adiga and M. Smitha, On maximum degree energy of a graph. Int. J. Contemp. Math. Sciences, Vol. 4, No. 8 (2009), 385 - 396

[3] T. Aleksić, Upper bounds for Laplacian energy of graphs. MATCH Commun. Math. Comput. Chem. 60 (2008) 435-439. 
[4] R. B .Bapat, S. Pati, Energy of a graph is never an odd integer. Bulletin of Kerala Mathematics Association 1(2): 129-132 (2004).

[5] V. Consonni, R. Todeschini, New spectral index for molecule description. MATCH Commun. Math. Comput. Chem. 60, 3 - 14 (2008).

[6] N. N. M. de Abreu, C. T. M. Vinagre, A. S. Bonifácio, I.Gutman, The Laplacian energy of some Laplacian integral graphs. MATCH Commun. Math. Comput. Chem. 60, 447 - 460 (2008)

[7] G. H. Fath-Tabar, A. R. Ashrafi, I. Gutman, Note on Laplacian energy of graphs. Bull. Acad. Serbe Sci. Arts (Cl. Math. Natur.) 137, 1-10 (2008)

[8] M. Fiedler, Additive compound matrices and an inequality for eigenvalues of symmetric stochastic matrices, Czech. Math. J., 24(99) 392-402 (1974).

[9] I. Gutman, The energy of a graph. Ber. Math-Satist. Sekt. Forschungsz. Graz 103, 1-22 (1978)

[10] I. Gutman, B. Zhou, Laplacian energy of a graph. Lin. Algebra Appl. 414, 29 - 37 (2006)

[11] I. Gutman, The Energy of a Graph: Old and New Results, ed. by A. Betten, A. Kohnert, R. Laue, A. Wassermann. Algebraic Combinatorics and Applications. (Springer, Berlin, 2001), pp. 196 - 211

[12] I. Gutman, X. Li, J. Zhang, in Graph energy, ed. by M. Dehmer, F. EmmertStreib. Analysis of Complex Networks. From Biology to Linguistics( WileyVCH, Weinheim, 2009), pp. 145 - 174

[13] I. Gutman, N. M. M. de Abreu, C. T. M. Vinagre, A. S. Bonifácio, S. Radenković, Relation between energy and Laplacian energy. MATCH Commun. Math. Comput. Chem. 59, 343-354 (2008)

[14] J. Liu, B. Liu, On relation between energy and Laplacian energy. MATCH Commun. Math. Comput. Chem. 61, 403-406 (2009)

[15] M. R. Rajesh Kanna, B. N. Dharmendra and R. Pradeep Kumar, Minimum Covering Distance Energy of a Graph. Applied Mathematical Sciences, Vol. 7, No. 111, (2013), 5525 - 5536.[ http://dx.doi.org/10.12988/ams.2013.38477] 
[16] M. R. Rajesh Kanna, B. N. Dharmendra, R. Shashi and R. A. Ramyashree, Maximum degree energy of certain mesh derived networks. International Journal of Computer Applications, 78 No.8 (2013) 3844.[http://dx.doi.org/10.5120/13513-1289]

[17] M. R. Rajesh Kanna, B. N. Dharmendra, G. Sridhara, The Minimum Dominating Energy Of A Graph. International Journal of Pure and Applied Mathematics $\mathbf{8 5}$ No. 4, 707-718 (2013)[http://dx.doi.org/10.12732/ijpam.v85i4.7]

[18] B. J. McClelland, Properties of the latent roots of a matrix: The estimation of pi-electron energies. J. Chem. Phys. 54, 640 - 643 (1971)

[19] S. Radenković, I. Gutman, Total $\pi$-electron energy and Laplacian energy: How far the analogy goes? J. Serb. Chem. Soc. 72, 1343-1350 (2007)

[20] M. Robbiano, R. Jiménez, Applications of a theorem by Ky Fan in the theory of Laplacian energy of graphs. MATCH Commun. Math. Comput. Chem. 62, 537-552 (2009)

[21] D. Stevanović, I. Stanković, M. Milošević, More on the relation between energy and Laplacian energy of graphs. MATCH Commun. Math. Comput. Chem. 61, 395-401 (2009)

[22] H. Wang, H. Hua, Note on Laplacian energy of graphs. MATCH Commun. Math. Comput. Chem. 59, 373-380 (2008)

[23] B. Zhou, New upper bounds for Laplacian energy. MATCH Commun. Math. Comput. Chem. 62, 553-560 (2009)

[24] B. Zhou, I. Gutman, Nordhaus-Gaddum-type relations for the energy and Laplacian energy of graphs. Bull. Acad. Serbe Sci. Arts (Cl. Math. Natur.) 134, 1-11 (2007)

[25] B. Zhou, On the sum of powers of the Laplacian eigenvalues of graphs. Lin. Algebra Appl. 429, 2239-2246 (2008)

[26] B. Zhou, I. Gutman, T. Aleksić, A note on Laplacian energy of graphs. MATCH Commun. Math. Comput. Chem. 60, 441-446 (2008)

[27] B. Zhou, I. Gutman, On Laplacian energy of graphs. MATCH Commun. Math. Comput. Chem. 57, 211-220 (2007) 
